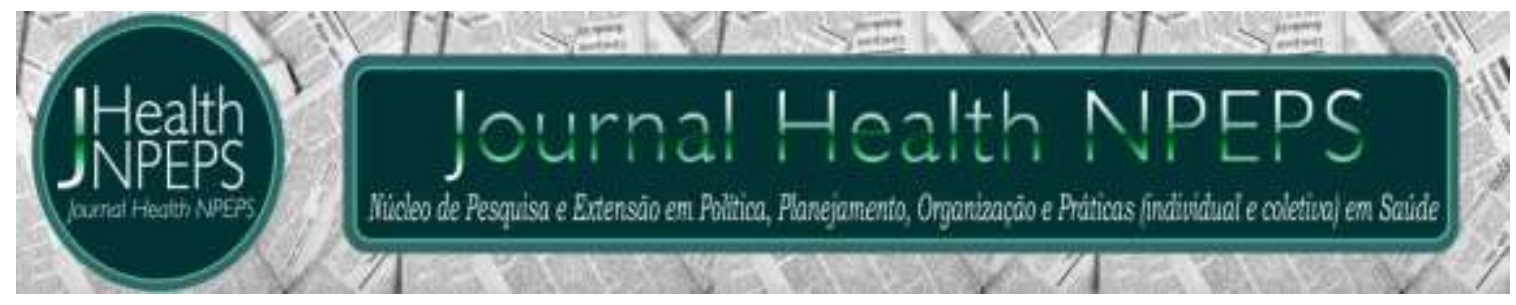

http://dx.doi.org/10.30681/252610104307

ARTIGO ORIGINAL

\title{
Uso de bebidas alcoólicas por estudantes de medicina
}

\author{
Use of alcoholic drinks by medicine students
}

Uso de bebidas alcohólicas por estudiantes de medicina

\section{Izete Soares da Silva Dantas Pereira ${ }^{1}$, Rodrigo Alves de Melo², Alison Jesissai Pinto Gurgel ${ }^{3}$, Ana Gabriela Miranda Barbosa ${ }^{4}$, Indira Coan Zanatta ${ }^{5}$, Sarah Lis Araújo Melo ${ }^{6}$, João Dantas Pereira ${ }^{7}$}

\section{RESUMO}

Objetivo: identificar a frequência do consumo de álcool por estudantes do curso de medicina. Método: estudo transversal, com abordagem quantitativa. Utilizou-se para a coleta de dados o questionário AUDIT aplicado a 128 estudantes que frequentam do primeiro ao oitavo períodos do curso de graduação em medicina. Resultados: a maioria não apresentou consumo elevado de álcool. 0 uso foi referido apenas em ocasiões festivas, sem que isso acarretasse consequências negativas na rotina dos acadêmicos. Não se verificou relação das variáveis sociodemográficas com a quantidade e intensidade do consumo de bebidas alcoólicas. Considerações finais: Apesar de não constituir ainda um problema na realidade dos participantes, torna-se de fundamental importância investir em ações/orientações preventivas sobre os riscos do uso de bebidas alcoólicas e os malefícios que podem trazer para o exercício desses futuros profissionais.

Descritores: Bebidas Alcoólicas; Estudantes de Medicina; Prevenção.

\footnotetext{
${ }^{1}$ Assistente social/Bacharel em Direito. Doutora em Saúde Pública. Docente do curso de Medicina da Universidade do Estado do Rio Grande do Norte (UERN). Natal, Rio Grande do Norte, Brasil. E-mail: izetedantas@hotmail.com ORCID ID: https://orcid.org/0000-0002-2239-6582 Autor principal - Endereço para correspondência: Rua das Tílias, 2114, Cidade Satélite-Pitimbú-Natal, RN, Brasil. CEP 59067-690.

${ }^{2}$ Acadêmico de Medicina. Universidade do Estado do Rio Grande do Norte (UERN). Mossoró, Rio Grande do Norte, Brasil. E-mail: rodrigoamelo3@gmail.com ORCID ID: https://orcid.org/0000-0002-8070-6614

${ }^{3}$ Acadêmico de Medicina. Universidade do Estado do Rio Grande do Norte (UERN). Mossoró, Rio Grande do Norte, Brasil. E-mail: ajesissai@gmail.com ORCID ID: https://orcid.org/0000-0003-1105-2407

${ }^{4}$ Acadêmica de Medicina. Universidade do Estado do Rio Grande do Norte (UERN). Mossoró, Rio Grande do Norte, Brasil. E-mail: belinha0623@gmail.com ORCID ID: https://orcid.org/0000-0002-5130-8086

${ }^{5}$ Acadêmica de Medicina. Universidade do Estado do Rio Grande do Norte (UERN). Mossoró, Rio Grande do Norte, Brasil. E-mail: indiracoan@hotmail.com ORCID ID: https://orcid.org/0000-0002-4884-1381

${ }^{6}$ Acadêmica de Medicina. Universidade do Estado do Rio Grande do Norte (UERN). Mossoró, Rio Grande do Norte, Brasil. E-mail: sarah.lis.araujo@hotmail.com ORCID ID: https://orcid.org/0000-0001-8238-9993

${ }^{7}$ Assistente Social/Bacharel em Direito. Doutor em Sociologia. Docente do curso de Direito da Universidade Federal do Rio Grande do Norte (UFRN). Natal, Rio Grande do Norte, Brasil. E-mail: jotarn@hotmail.com ORCID ID: https://orcid.org/0000-0001-9855-827X
}

Este artigo está licenciado sob forma de uma licença Creative Commons Atribuição 4.0 Internacional, que permite uso irrestrito, distribuição e reprodução em qualquer meio, desde que a publicação original seja corretamente citada. 


\begin{abstract}
Objective: to identify the frequency of alcohol consumption by medical students. Method: cross-sectional study with a quantitative approach. The AUDIT questionnaire applied to 128 students attending the first to eighth periods of the undergraduate medical course was used for data collection. Results: most did not have a high alcohol consumption. The use was mentioned only on festive occasions, without this having negative consequences on the academic routine. There was no relationship between sociodemographic variables and the amount and intensity of alcohol consumption. Final considerations: although it is not yet a problem in the reality of the participants, it is of fundamental importance to invest in preventive actions / guidance on the risks of using alcoholic beverages and the harm they can bring to the exercise of these future professionals.
\end{abstract}

Descriptors: Alcoholic beverage; Medicine Students; Prevention.

\title{
RESUMEN
}

Objetivo: identificar la frecuencia del consumo de alcohol por parte de los estudiantes de medicina. Método: estudio transversal con enfoque cuantitativo. El cuestionario AUDIT aplicado a 128 estudiantes que asistieron a los períodos primero a octavo del curso de medicina de pregrado se utilizó para la recopilación de datos. Resultados: la mayoría no tenían un alto consumo de alcohol. El uso se mencionó solo en ocasiones festivas, sin que esto tenga consecuencias negativas en la rutina académica. No hubo relación entre las variables sociodemográficas y la cantidad e intensidad del consumo de alcohol. Consideraciones finales: Aunque todavía no es un problema en la realidad de los participantes, es de fundamental importancia invertir en acciones / pautas preventivas sobre los riesgos del uso de bebidas alcohólicas y el daño que pueden ocasionar al ejercicio de estos futuros profesionales.

Descriptores: Bebidas Alcohólicas; Estudiantes de Medicina; Prevención.

\section{INTRODUÇÃO}

0 consumo de drogas, sejam elas lícitas ou ilícitas, tem aumentado nas últimas décadas, se caracterizando como uma questão de saúde pública no mundo ${ }^{1}$. 0 referido consumo é responsável por cerca de $4 \%$ das mortes, $5 \%$ dos casos de doenças e $90 \%$ das internações hospitalares por dependência química e outras comorbidades. 0 uso de drogas pode acarretar inúmeros prejuízos ao indivíduo e à sociedade, gerando problemas políticos, econômicos e sociais, afetando a qualidade de vida de todos os envolvidos ${ }^{2-4}$.

Entre maio e outubro de 2015 foi realizada uma pesquisa em todo o Brasil com cerca de 17 mil pessoas com idades entre os 12 e os 65 anos, tendo como objetivo estimar e avaliar os parâmetros epidemiológicos do uso 
de drogas. Os resultados revelaram que $3,2 \%$ dos brasileiros usaram drogas ilícitas nos 12 meses anteriores à pesquisa. Esse dado equivale a 4,9 milhões de pessoas, sendo maior entre homens $(5 \%)$ e menor nas mulheres $(1,5 \%)^{3}$.

O impacto que o uso de álcool tem na saúde das pessoas tende a ser cada vez maior pelo fato de ser uma droga lícita, de custo reduzido, de fácil acesso e ter como suporte campanhas publicitárias que estimulam o seu consumo. Observa-se um aumento significativo nas diferentes classes sociais e faixas etárias com iniciação nestas últimas, cada vez mais precoce ${ }^{2,4}$.

Em 2015, a Organização Mundial da Saúde (OMS) fez uma projeção, por país, sobre o consumo de álcool puro e a estimativa para o Brasil fora de 9,1 litros por pessoa com 15 anos ou mais. No país, cerca de $70 \%$ da população faz uso das bebidas alcoólicas ${ }^{1}$. Segundo essa instituição, o álcool é a substância psicoativa mais consumida no mundo (2 bilhões de pessoas). Nesse contexto, há um público representado pelos jovens universitários. Há diferenças nos problemas decorrentes do consumo de álcool entre adultos e jovens. Nesses se constatam mais complicações relacionadas às interações com professores, colegas e famíliares ${ }^{3,5,6}$.

0 consumo afeta o rendimento acadêmico desses estudantes de forma negativa levando a ausência às aulas e avaliações, desconcentração durante as atividades acadêmicas, problemas de relacionamentos com professores, colegas e familiares. Ocasiona episódios de agressividade, níveis baixos de convívio social, risco de gravidez indesejável e doenças sexualmente transmissíveis, além de incitar a violência doméstica e no trânsito, provocando lesões e acidentes $^{7-10}$. 0 uso abusivo de álcool e o alcoolismo, por exemplo, são, direta e indiretamente pelo surgimento de danos físicos, mentais e sociais $^{11}$.

A expressão, alcoolismo tem sido empregada para designar o consumo regular e inadequado de bebidas alcoólicas ou o conjunto de consequências relacionadas ao consumo excessivo e prolongado do álcool, vontade incontrolável de beber, falta de controle ao tentar parar a ingestão, tolerância (doses cada vez maiores para sentir os mesmos efeitos da bebida) ${ }^{11}$. A 
dependência física é uma manifestação de sintomas físicos e psíquicos nas situações de abstinência alcoólica. 0 entendimento sobre a etiologia do alcoolismo é amplo, incluindo fatores biológicos, psicológicos e sociais $3-5,10,11$.

Para fazer frente a essa realidade em algumas universidades já existem projeto ou programa de prevenção e/ou orientação para os estudantes envolvidos com o consumo problemático de bebidas alcoólicas ${ }^{11}$. Os jovens universitários são considerados mais vulneráveis ao consumo de bebidas alcoólicas, sendo estes uma das maiores preocupações referentes à saúde e ao comportamento do estudante cuja prática pode acarretar $\mathrm{O}$ alcoolismo9,12,13.

Para o que interessa reter desta pesquisa sobre o uso do álcool entre estudantes de medicina são elucidativos os dados de um levantamento nacional referente ao uso de drogas por universitários brasileiros de instituições públicas e privadas, em todas as 27 capitais do país ${ }^{11}$. Esse estudo nacional apresentou os seguintes resultados: o álcool é a substância mais consumida nos últimos 12 meses. Além disso, 80\% do público universitário entrevistado na pesquisa alegaram já ter consumido algum tipo de bebida alcoólica e $1 / 3$ afirmou já ter realizado o consumo em binge em pelo menos uma ocasião.

Relativamente ao álcool são identificados quatro padrões de consumo: 1) o moderado, sem risco; 2) o arriscado, que tem o potencial de produzir danos; 3 ) o nocivo, que se define por um padrão constante de uso associado a danos à saúde e 4) o consumo em binge, que diz respeito ao uso eventual de álcool em grande quantidade ${ }^{14,15,16}$. $O$ ato do binge drinking vem crescendo no âmbito estudantil. 0 termo é utilizado para designar episódios nos quais há um consumo excessivo de bebida alcoólica. A quantidade que corresponde a esta prática seria de cinco ou mais doses de bebidas alcoólicas em uma única ocasião (no caso dos homens) ou quatro ou mais (para mulheres), sendo irrelevante a frequência dos episódios. A relevância dessa abordagem se prende ao fato de ser essa prática muito utilizada como "trote" para os calouros, provocando, muitas vezes, coma alcoólico e já ter levado à morte em casos graves ${ }^{14,17,18}$. 
Dentre as variáveis sócio culturais, a prática religiosa é um fator importante em relação à prevenção do alcoolismo. Estudo envolvendo jovens mostrou que a religião é um fator protetor ao consumo de drogas lícitas e ilícitas e ajuda o ser humano a se autoconhecer, amenizando as angústias e dramas da vida e comportamentos degradantes. Os indivíduos com uma menor taxa de espiritualidade têm 3,3 vezes mais risco de um consumo de álcool problemático. Essa variável, a religião, pode agir como fator protetor para o uso de drogas entre estudantes adolescentes e jovens, possivelmente em razão das normas de conduta disseminadas pela maioria das religiões ${ }^{19}$.

Ao ingressar na universidade muitos estudantes vivenciam novas experiências como se distanciar da família, residir com outros estudantes e passar a maior parte do tempo no ambiente universitário, além de adquirirem maior liberdade e independência para a tomada de decisões. Essas novas experiências podem favorecer um maior consumo de álcool, assim como os riscos associados a tal consumo ${ }^{2,5}$.
0 ingresso na universidade tem sido considerado um período crítico, principalmente para os calouros, com maior vulnerabilidade para o início e manutenção do consumo de álcool e outras drogas. Por outro lado, os calouros apresentam um risco maior de vivenciar as consequências relacionadas ao consumo de álcool. Nesse sentido, quanto mais tarde for 0 início do consumo de álcool, menores serão as probabilidades de se desenvolver problemas decorrentes desse comportamento, incluindo níveis de consumo abusivo ou, até mesmo, uma dependência ${ }^{20}$.

Alguns fatores estão relacionados com o consumo de álcool entre os estudantes como, por exemplo, ingresso precoce na universidade, área de formação universitária (humanas, tecnológico, biomédica, etc.), distância da família, falta de maturidade emocional, facilidade de acesso ao álcool, entre outros $^{6,17,21}$.

Além dos fatores já mencionados, no caso dos estudantes de medicina, um dos fatores que contribuem para a iniciação e o uso constante e/ou abusivo de álcool é a grande exigência do rendimento 
acadêmico. A competitividade existente entre eles, as extensas e cansativas práticas acadêmicas, longas horas de dedicação exclusiva ao curso, também devem ser consideradas. Há uma quantidade excessiva de conteúdos que se traduz numa carga horária que, em muitas faculdades, ultrapassam as 9.000 horas. Além disso, tem-se a cobrança por um bom desempenho estudantil, culminando numa preocupação diária em conseguir notas adequadas para aprovação. Todos esses representam fatores estressores na rotina desses estudantes, o qual pode utilizar o consumo de álcool com o intuito de enfrentar os obstáculos existentes $7,8,18,22,23$.

Frente a esse cenário, as questões relacionadas ao consumo de álcool têm-se configurado como como um problema de saúde pública, visto a sua magnitude, vulnerabilidade $e$ transcendência. Esse último termo é aqui entendido como o custo pessoal e social dos agravos à população, em que tudo que se relaciona ao processo saúde-doença-intervenção afeta diretamente as relações sociais, econômicas, profissionais e culturais ${ }^{20}$.
Do exposto, as universidades que registram baixo consumo de bebidas alcoólicas podem se constituir em espaços sociais estratégicos para a promoção de saúde desses grupos específicos, com consequente impacto positivo sobre a população em geral. Essa promoção da saúde no âmbito acadêmico implica em proporcionar condições necessárias aos estudantes para melhorar e exercer o controle sobre sua própria saúde ${ }^{15}$.

A preocupação com a temática motivou o presente estudo que tem como objetivo identificar a frequência do consumo de álcool por estudantes do curso de medicina.

\section{MÉTODO}

Tratou-se de um estudo transversal, com abordagem quantitativa, realizada com 128 acadêmicos do $1^{\circ}, 2^{\circ}, 3^{\circ}, 5^{\circ}$ e $7^{\circ}$ do curso de graduação em medicina de uma Universidade do Estado do Rio Grande do Norte. A escolha desse local ocorreu por ser uma Universidade pública e pela oportunidade da pesquisa em abordar uma temática ainda não estudada, nesse lócus. 
No momento da pesquisa, o curso contava com 196 estudantes $(100 \%)$, regularmente matriculados do $1^{\circ}$ ao ${ }^{\circ} 12^{\circ}$ períodos. No entanto, os estudantes do $4^{\circ}, 6^{\circ}$ e $8^{\circ}$ períodos não participaram da pesquisa, devido à incompatibilidade

e/ou indisponibilidade das respectivas turmas. Os estudantes do $9^{\circ}$ ao $12^{\circ}$ estavam no internato, o que dificultou o contato com os mesmos.

Assim sendo, do total previsto, ou seja, o universo, apenas 128 $(65,3 \%)$ acadêmicos, de ambos os sexos, responderam aos instrumentos.

De acordo com os critérios estabelecidos para este estudo, foram incluídos todos os estudantes regularmente matriculados no $1^{\circ}$ ao $12^{\circ}$ período do curso de medicina e excluídos aqueles que frequentavam disciplinas especiais no curso de medicina, bem como os da PósGraduação e os acadêmicos que por algum motivo não estiveram presente no momento da coleta de dados, como, por exemplo, por estarem afastados da sala de aula por licença médica/maternidade.

Os dados foram coletados nas salas de aula teóricas durante o primeiro semestre de 2018, antes do início ou após o término, sem a presença do professor. Utilizou-se para a coleta de dados o questionário AUDIT (Alcohol Use Disorders Identification Test) e um formulário de coleta de informações elaborado pelos pesquisadores contendo informações sociodemográficas: sexo, faixa etária, estado civil, procedência, vínculo laboral e o semestre em que estavam matriculados. O tempo de aplicação do instrumento variou de 10 a 20 minutos, dependendo do número de estudantes na turma.

O AUDIT é um modelo de questionário desenvolvido pela Organização Mundial de Saúde (OMS), adaptado para uso no Brasil. Esse instrumento inclui perguntas sobre as consequências do consumo, quantidade e frequência de ingestão de álcool. Tem como característica ser um recurso autoaplicável e de fácil preenchimento, não requisitando um treinamento prévio especializado. É composto por 10 itens capazes de avaliar o consumo de álcool nos últimos 12 meses. Cada um dos itens tem 5 respostas possíveis, pontuadas de 0 a 4 (exceto os itens 9 e 10, que pontuam apenas 0,1 e 2 ), variando $o$ escore total de 0 a 36. De acordo com o resultado obtido, é possível 
classificar o respondente dentro de 4 padrões de consumo de álcool ou zonas de risco, sendo elas: 1) uso de baixo risco (escore de 0 a 7 pontos); 2) uso de risco ( 8 a 15 pontos); 3) risco nocivo (16 a 19 pontos) e 4) provável dependência (20 ou mais pontos) $)^{15,19,24}$.

Após a coleta, os dados foram armazenados em planilhas do Excel versão 20.0 para posterior processamento. Para a análise, utilizou-se a estatística descritiva com frequências absolutas e relativas, apresentados em forma de gráfico e tabela.

A pesquisa foi submetida ao Comitê de Ética em Pesquisa da Universidade do Estado do Rio Grande do Norte-CEP/UERN, sob o número CAAE 25736619.7.0000.5294 e aprovada pelo Parecer $\mathrm{n}^{\circ} \quad$ 1.981.033. Foram respeitados, rigorosamente, todos os princípios e as diretrizes éticas de pesquisa envolvendo seres humanos, em atendimento à Resolução 466/2012 do Conselho Nacional de Saúde e suas complementares. Todos os estudantes que aceitaram participar do estudo assinaram 0 Termo de Consentimento Livre e Esclarecido (TCLE).

\section{RESULTADOS E DISCUSSÃO}

Os dados a seguir apresentados incluem as informações sobre o perfil sociodemográfico dos acadêmicos e os resultados dos questionários (AUDIT) aplicados.

Os acadêmicos de medicina estiveram distribuídos nos seguintes períodos: $21,0 \%$ do primeiro; $24,0 \%$ do segundo; $15,0 \%$ do terceiro; $24,0 \%$ do quinto e $16,0 \%$ do sétimo, totalizando 128 estudantes $(65,3 \%)$.

$\mathrm{Na}$ Tabela 1, observa-se que do total de estudantes do curso de medicina que participaram deste estudo, $56,25 \%$ são do sexo feminino. A faixa etária predominante foi representada por aqueles com idade compreendida entre os 18 e os 25 anos. Em relação ao estado civil $92,97 \%$ são solteiros. A maioria, $82,03 \%$, é procedente de outros estados da federação. Quanto ao quesito ocupação, apenas, 7,03\% desempenham alguma atividade remunerada. Os demais se dedicam, exclusivamente, aos estudos. 
Tabela 1 - Perfil sociodemográfico dos estudantes. Mossoró - RN, Brasil. 2018.

\begin{tabular}{lrr} 
& N & $\%$ \\
\hline Sexo & 72 & \\
Feminino & 56 & 56,25 \\
Masculino & & 43,75 \\
Faixa etária & 96 & 75,00 \\
18 a 25 anos & 30 & 23,44 \\
25 a 35 anos & 01 & 0,78 \\
35 a 45 anos & 01 & 0,78 \\
45 a 55 anos & 119 & \\
Estado civil & 09 & 92,97 \\
Solteiro & 23 & 7,03 \\
Casado & 105 & 19,97 \\
Procedência & & 82,03 \\
Mossoró & 09 & \\
Outros estados & 119 & 7,03 \\
Ocupação & 128 & 92,97 \\
Sim & & 100,00 \\
Não & & \\
Total & & \\
\hline
\end{tabular}

A associação de variáveis sociodemográficos com o consumo de bebidas alcoólicas tem sido considerada em vários estudos realizados no Brasil e em outros países. Estudos têm mostrado que a quantidade e o padrão de consumo de álcool variam na população de acordo com idade, etnia/cor da pele, condição socioeconômica e/ou educação, trabalho, estado civil e características da vizinhança, sendo que essas podem atuar de maneira diferente entre homens e mulheres e apresentar resultados divergentes $2,6,14,15,18,25$.

$03^{\circ}$ Levantamento Nacional sobre o uso de drogas pela população brasileira destacou que mais de um milhão de adolescentes e jovens, na faixa etária dos 18 aos 34 anos, consumiram álcool e tabaco nos doze meses anteriores à pesquisa. Desses, cerca de 400 mil fizeram uso do álcool e, pelo menos, uma substância ilícita. Em relação ao sexo, a dependência do álcool foi 3,4 vezes mais frequente entre os homens do que entre as mulheres, no mesmo período. Contudo, as mulheres apresentaram prevalência superior à dos homens no consumo de álcool quando aliado a, pelo menos, um medicamento não prescrito. O uso de álcool associado a uma droga ilícita ou a um medicamento não prescrito pareceu estar diretamente relacionado ao nível de escolaridade, ou seja, à medida que o nível de escolaridade se 
eleva, são maiores as prevalências de consumo múltiplo dessas drogas ${ }^{3}$.

Esses resultados sinalizam no sentido de que o consumo de drogas, entre elas o álcool, está disseminado na população jovem e que independe do gênero.

Os resultados apresentados pela Pesquisa Nacional de Saúde realizada em 2013 corroboram que uma união estável constitui um dos fatores protetores/inibidores do consumo de álcool entre as mulheres ${ }^{6}$. Estudo desenvolvido na Colômbia mostrou que o consumo de álcool pelos jovens universitários era maior entre as pessoas solteiras que se encontram nos primeiros anos da faculdade. Nos homens, essa conduta vai se mantendo ao longo do curso e aumentando a prevalência ${ }^{19}$.

Neste estudo a procedência dos participantes é majoritariamente de outros estados da federação, o que significa que moram sozinhos, com amigos ou ainda em pensionatos, longe de casa, sem o apoio da família.

Diversas pesquisas realizadas no Brasil entre estudantes mostraram que morar sozinho ou com amigos, longe da vigilância dos pais ou de outros familiares pode aumentar, consideravelmente, os riscos para o consumo de álcool e outras drogas. Um ambiente de maior liberdade pode facilitar o acesso e o consumo dessas drogas $^{2,21,23}$.

Em relação à variável ocupação, a Pesquisa Nacional de Saúde (2013), mostrou que os estudantes que tinham algum vínculo laboral apresentaram um percentual de baixo risco para o consumo de bebidas alcoólicas quando comparados àqueles que não exerciam atividade laboral ${ }^{6}$.

No que diz respeito à frequência com que os estudantes de medicina consomem bebidas alcoólicas, neste estudo, 33,60\% afirmaram ser abstêmios, enquanto $66,41 \%$ referiram beber de uma a quatro vezes por mês (Tabela 2). 0 valor identificado nesta pesquisa é semelhante aos números encontrados em um estudo realizado na capital do Distrito Federal, onde $33,60 \%$ dos participantes também se declararam abstêmios ${ }^{9}$ Esse percentual representa um valor, consideravelmente, inferior ao de outros estudos feitos entre estudantes de cursos universitários no Brasil, nos quais a prevalência do consumo de álcool variou de 75 a $93,7 \% 2,7,8,18,21$. 
Tabela 2 - Consumo de bebidas alcoólicas pelos estudantes de medicina. Mossoró - RN, Brasil. 2018. $(n=128)$

\begin{tabular}{lrr}
\hline Consumo & N & \multicolumn{1}{c}{$(\%)$} \\
\hline Nunca & 43 & 33,60 \\
Uma vez por mês ou menos & 49 & 38,28 \\
Duas a quatro vezes por mês & 34 & 26,56 \\
Duas a três vezes por semana & 02 & 1,56 \\
Total & $\mathbf{1 2 8}$ & $\mathbf{1 0 0 , 0 0}$ \\
\hline
\end{tabular}

Ao comparar uma pesquisa realizada sobre o uso de drogas entre estudantes de medicina com universitários de outros cursos, os resultados mostraram que aqueles consumiam mais bebidas alcoólicas do que os dos outros cursos ${ }^{25}$. Portanto, mesmo conhecendo os danos potenciais e diretos que essa substância pode causar ao próprio organismo e à sociedade em geral, os estudantes de Medicina ainda buscam alguma utilidade no uso dessa droga, ficando sujeitos e vulneráveis às consequências desse consumo ${ }^{26}$.

A literatura consultada mostrou que essa não é apenas a realidade do Brasil. Um estudo similar realizado na Universidade da Cantábria (Espanha) encontrou um padrão de consumo do álcool de pouco mais da metade da população entre acadêmicos de medicina $e$ enfermagem ${ }^{17}$.

Na Finlândia, realizou-se uma pesquisa para conhecer a relação entre desempenho acadêmico e consumo de álcool entre os alunos de sete faculdades da Universidade de Turku, envolvendo 1177 estudantes de graduação. Os resultados mostraram que os alunos relataram um consumo de longa duração e grande quantidade de bebida em torno de 50\%, alta frequência de bebida (41\%), consumo episódico pesado em 66\%, problema de consumo de álcool (29\%) e possível dependência de álcool para $9 \%$ dos participantes $^{27}$.

A preocupação com o uso nocivo de álcool por parte da população estudantil nos vários países ganhou destaque no relatório da OMS que apresentou resultados de estudos americanos, mostrando que $2 / 3$ da população de universitários dos Estados Unidos consumiam álcool e que cerca de metade o fazia em um padrão excessivo ${ }^{1}$. Apesar de ser lícito, o consumo de álcool pode ser uma "porta de entrada" para as drogas ilícitas. Além disso, o uso 
associado do álcool com drogas ilícitas pode intensificar os efeitos deletérios dessas drogas quando comparado ao consumo isolado ${ }^{20,28,29}$.

$\mathrm{Na}$ Etiópia um estudo transversal realizado entre 725 estudantes universitários em 2015, constatou o uso problemático de álcool esteve associado à fobia social, baixo desempenho acadêmico, uso na vida e uso de outras drogas ${ }^{30}$.

As bebidas alcoólicas foram aquelas que em vários estudos apresentaram um maior número de usuários em todos os níveis de risco, independentemente dos instrumentos de coleta de dados utilizados e dos tipos de drogas lícitas e/ou ilícitas consumidas $^{1,5,11,23}$. A prevenção e a redução do uso nocivo do álcool devem ser tratadas como prioridade, para que seja possível alcançar a meta previamente estipulada de redução relativa de $10 \%$ no consumo nocivo mundial de álcool em 2025, segundo a OMS ${ }^{1}$.

A relação entre o uso de bebida alcoólica e o sexo apresentado na tabela 3, mostra que neste estudo houve um ligeiro predomínio do sexo feminino em relação ao masculino, o que vem divergindo da maior parte dos estudos, onde essa relação (mulheres vs consumo de álcool) não é estatisticamente significativa. 0 uso do álcool tem sido mais frequente entre os estudantes do sexo masculino, embora esse aspecto não seja consensual entre os autores ${ }^{2,10,15}$.

Tabela 3- Relação entre gênero e risco de acordo com a classificação AUDIT. Mossoró - RN, 2018. $(n=128)$

\begin{tabular}{lccccr}
\hline Sexo & Risco I & Risco II & Risco III & Risco IV & Total \\
\hline Feminino & 63 & 07 & 02 & 00 & 72 \\
Masculino & 47 & 08 & 01 & 00 & 56 \\
Total & 110 & 15 & 03 & 00 & 128 \\
\hline
\end{tabular}

Ainda em relação ao sexo, o II Levantamento Nacional de Álcool e Drogas - 2012 comparou resultados do consumo de álcool entre homens e mulheres em dois períodos, 2006 e 2012. No ano de 2006, mulheres que apresentavam uso regular (um ou mais vezes por semana) de consumo de álcool foram $27 \%$, aumentando para $38 \%$ em 2012, enquanto que os homens aumentaram de $54 \%$ para $63 \%{ }^{13}$. Esses números chamam atenção 
para um crescimento do etilismo no sexo feminino, hábito que, em décadas passadas, era observado apenas no público masculino ${ }^{3}$.

Os resultados de um estudo realizado com estudantes do curso de medicina de uma Universidade do Sul Fluminense, utilizando a mesma metodologia do AUDIT mostrou que o hábito de beber no primeiro semestre, em ambos os gêneros, aumentou com o decorrer do curso. A prática de beber pesado foi superior nos homens em comparação às mulheres ${ }^{28}$.

Embora não se enquadre nos critérios de dependência, existe uma probabilidade maior das pessoas que fazem uso de consumo nocivo de álcool não cumprirem as tarefas habituais e de se envolverem em atos de violência ${ }^{17}$.

Outros estudos nacionais e internacionais concluíram que pessoas que aderem a comportamentos saudáveis apresentam maior longevidade, redução da vulnerabilidade ao adoecer e da morbimortalidade geral, além da diminuição do impacto financeiro sobre o sistema de saúde e de efeitos adversos em sua qualidade de vida $^{15,17,19,20,23,27,30,31}$.
Um estudo realizado na Colômbia mostrou que para cada oito homens que consomem bebidas alcoólicas há três mulheres ${ }^{19}$. Têm-se observado que nos últimos anos, no Brasil, registra-se uma tendência gradual, por parte das mulheres, em aumentar o consumo e de forma mais precoce $^{3,11}$.

Foi realizado um estudo analítico transversal, utilizando teste de triagem de envolvimento de álcool, tabagismo e outras substâncias (ASSIST) em dois grupos populacionais com o objetivo de calcular a prevalência de risco de uso de substâncias psicoativas entre estudantes, professores e trabalhadores de uma universidade pública do Equador. Os resultados mostraram a prevalência de consumo de alto risco para álcool de 5,3\% para estudantes e $4,9 \%$ para trabalhadores e professores. O tabaco e a cannabis foi alta nos grupos estudados ${ }^{23}$.

Ao se comparar estudos realizados no Brasil com outros similares em países como a Espanha, Colômbia, Equador, Finlândia, Turquia já citados, anteriormente, que utilizaram método semelhante, constataram-se resultados parecidos no consumo de álcool pelos 
estudantes ${ }^{17,19,23,27,30}$. Naqueles países o consumo de álcool também é considerado alto com Riscos III e IV na escala AUDIT, ao contrário do que se verificou no presente estudo ${ }^{24}$.

Neste estudo, nenhum dos participantes, apresentou padrão de consumo associado ao nível IV, ou seja, indicativo de provável dependência de álcool. Esse critério de dependência pode ser constatado quando existe um padrão de uso em que a pessoa desenvolve mudanças fisiológicas, cognitivas

e
comportamentais como desejo intenso de consumir bebidas alcóolicas; falta de controle sobre o consumo; continuar consumindo apesar das consequências; dar maior prioridade ao consumo do que às outras atividades; aumento da tolerância e presença da síndrome de abstinência com a descontinuidade do consumo ${ }^{21}$.

Os autores são consensuais que as universidades precisam avaliar os problemas de bebida e distúrbios provocados pelo uso de álcool entre os estudantes. São necessárias medidas para definir e implementar estratégias de prevenção para reduzir os riscos decorrentes dessa prática. Os esforços de promoção da saúde podem se concentrar nas crenças e expectativas sobre o álcool e priorizar os grupos de estudantes em risco, envidando esforços mais eficientes ${ }^{15,20}$.

O uso problemático de álcool entre estudantes de medicina é preocupante, pois há a possibilidade desse uso permanecer na atuação profissional desses futuros médicos. Essa prática pode interferir na habilidade em fazer diagnóstico precoce, encaminhamento e tratamento de seus pacientes dependentes ou não do álcool $3,6,11,16,28$. Essa preocupação é agravada pelo fato de que o médico servirá de modelo para seus pacientes. Além disso, a ideia de que o médico apenas indicaria o caminho a ser seguido sem, necessariamente, o fazer é contraditória e pode tornar o tratamento com baixa adesão por parte dos pacientes. O consumo de bebidas alcoólicas, por parte de qualquer pessoa cria risco para dependência dessas substâncias, o que se caracteriza como um grande problema social e de saúde 3,6,11,16.

As consequências do uso de álcool, além de todo o impacto negativo para o indivíduo, família e amigos acabam por onerar a sociedade. De forma direta e indireta, potencializam os custos em hospitais e 
outros dispositivos do sistema de saúde, sistema judiciário, previdenciário, perda de produtividade do trabalho, absenteísmo, desemprego, entre outros. Em todo o mundo, nota-se que as faixas etárias mais jovens são as principais afetadas em relação às mortes associadas ao uso do álcool, traduzindo como uma maior perda de pessoas na fase mais produtiva em que estão economicamente ativas ${ }^{31}$.

\section{CONSIDERAÇÕES FINAIS}

A pesquisa mostrou que a maioria dos participantes faz uso de álcool esporadicamente, sendo considerado de baixo risco (Risco I) e um terço se declarou abstêmio. 0 consumo de bebidas alcoólicas ocorre de forma pontual, em festas ou comemorações, com frequência média de duas a quatro vezes por mês. Tal padrão de uso, configura-se como oportunidade para que o contexto em que esses estudantes estão inseridos, seja um espaço de promoção e prevenção de problemas e agravos à saúde, relacionados ao uso e abuso de álcool. Essa constatação é muito importante e positiva para a realidade estudada na medida em que se sabe, através da literatura, que o consumo de álcool e de outras drogas tem crescido no país, entre estudantes da área da saúde, sobretudo, entre os de medicina.

Apesar de ainda não se constituir um problema na realidade dos participantes, torna-se de fundamental importância investir em ações/orientações preventivas sobre riscos do uso de bebidas alcoólicas e os malefícios que podem trazer para o exercício desses futuros profissionais. Essas estratégias se tornam pertinentes, principalmente, pelo fato de esses indivíduos serem futuros especialistas na área da saúde e, portanto, profissionais que orientarão outras pessoas acerca deste assunto.

Como limitação da pesquisa, pode-se citar a ausência de alguns estudantes de outros períodos letivos durante a coleta dos dados. Isso pode estar relacionado ao fato de que a coleta ocorreu durante o período em que a instituição esteve com grande parte das suas atividades paralisadas por reivindicação salarial e melhores condições de trabalho dos servidores. Desta forma, salienta-se que o estudo pode ter subestimado a prevalência daqueles que fazem o consumo de álcool nas categorias de Risco III e IV. 
Outra limitação a ser considerada é o fato de que o formulário sociodemográfico utilizado não incluiu a religião como uma variável a ser identificada, uma vez que essa é um importante fator de proteção ao consumo de álcool e de outras drogas lícitas e ilícitas. Muitas pessoas que não fazem uso de bebidas alcóolicas associam essa decisão à sua prática religiosa.

Sugerem-se a realização de outros estudos, com metodologias quantitativas em que sejam avaliados os índices de consumo do álcool e sua associação com outras drogas entre acadêmicos de outros cursos e outras Instituições de Ensino Superior. Esses estudos poderão ampliar os conhecimentos sobre os padrões de uso dessas drogas.

\section{REFERÊNCIAS}

1. Organização Mundial da Saúde. Relatório Global sobre Álcool e Saúde 2018. Genebra, Suiça, 2018. Disponível em: Disponível em: https://cisa.org.br/index.php/pesq uisa/dados-oficiais. Acesso em: 12 jun 2019.

2. Ribeiro GFF, França VM, Faria RLBC, Cuellar PMG, Martins MLB.
Álcool: uso por estudantes de medicina da Universidade Federal do Tocantins. Rev Cereus. 2015; 7(1):26-39.

3. Bastos FIPM, Vasconcellos MT, De Boni RB, Reis NB, Coutinho CFS. $3^{\circ}$ Levantamento Nacional sobre o uso de drogas pela população brasileira. Rio de Janeiro: FIOCRUZ/ICICT; 2017.

4. Souza LGS, Menandro MCS, Menandro PRM. O alcoolismo, suas causas e tratamento nas representações sociais de profissionais de Saúde da Família. Physis. 2015; 25(4):1335-1360.

5. Vecchia MD. Notas sobre a conjuntura da política de drogas no Brasil. J Health NPEPS. 2018; 3(2):298-303.

6. Machado IE, Monteiro MG, Malta DC, Lana FCF. Pesquisa Nacional de Saúde 2013: relação entre uso de álcool e características sociodemográficas segundo o sexo no Brasil. Rev bras epidemiol. 2017; 20(3):408-422.

7. Pinheiro MA, Torres LF, Bezerra MS, Cavalcante RC, Alencar RD, Donato $A C$, et al. Prevalência e fatores associados ao consumo de álcool e tabaco entre estudantes de medicina no Nordeste do Brasil. Rev 
bras educ med. 2017; 41(2):231250.

8. Nascimento MI, Costa JS, Pereira MA, Kiepper MS, Keher NB, Moraes RFS. Uso de álcool por estudantes de medicina segundo características de cursos e escolas médicas: uma revisão da literatura. Rev bras educ med. 2019; 43(1 supl.):98-107.

9. Trindade BPA, Diniz AV, Sá-Júnior AR. Uso de drogas entre estudantes universitários: uma perspectiva nacional. Rev Med Saude Brasilia. 2018; 7(1):52-60.

10. Fernandes TF, Monteiro BMM, Silva JBM, Oliveira KM, Viana NAO, Gama CAP, et al. Uso de substâncias psicoativas entre universitários brasileiros: perfil epidemiológico, contextos de uso e limitações metodológicas dos estudos. Cad saúde colet. 2017; 25(4):498-507.

11. Brasil. Presidência da República. Secretaria Nacional de Políticas sobre Drogas. I Levantamento Nacional sobre o Uso de Álcool, Tabaco e Outras Drogas entre Universitários das 27 Capitais Brasileiras. Brasília: SENAD; 2010.

12. Oliveira SKM, Sousa ÁAD, Cavalcanti SL, Taveira MGMM,
Correia DS, Freitas DA. Uso de Bebidas Alcoólicas entre Acadêmicos da Área de Saúde. Rev Bras Educ Med. 2016; 40(3):44645.

13. Laranjeira R, Madruga CS, Pinsky I, Caetano R, Ribeiro $M$, Mitsuhiro S. II Levantamento Nacional de Álcool e DrogasConsumo de Álcool no Brasil: Tendências entre 2006/2012. São Paulo: INPAD; 2013.

14. Pelicioli M, Barelli C, Gonçalves CBC, Hahn S, Scherer JI. Perfil do consumo de álcool e prática do beber pesado episódico entre universitários brasileiros da área da saúde. J Bras Psiquiatr. 2017; 66(3):150-156.

15. Raposo JCS, Costa ACQ, Valença PAM, Zarzar PM, Diniz AS, Colares $\mathrm{V}$, et al. Uso de drogas ilícitas e binge drinking entre estudantes adolescentes. Rev Saúde Pública. 2017; 4(51):51-83.

16. Mendonça AKRH, Nogueira MSN, Andrade RLB, Azevedo DX, Jesus CVF, Lima SO. Binge drinking entre estudantes de medicina de universidades particular e pública de um estado do nordeste brasileiro. Temas saúde. 2018; 18(3):30-53. 
17. Herrero-Montes M, Alonso-Blanco C, Paz-Zulueta M, Sarabia-Cobo C, Ruiz-Azcona L, Parás-Bravo P. Binge Drinking in Spanish University Students: Associated Factors and Repercussions: A Preliminary Study. Int J Environ Res Public Health. 2019; 16:4822.

18. Maia DAM, Marques RB, Maia Filho AM. Consumo de bebidas alcoólicas e a prática do binge drinking em acadêmicos de medicina. Rev Interdisciplin. 2017; 10(1):139-146.

19. Yañez-Castillo BG, VIllar-Luis MA, Alonso-Castillo MM. Espiritualidad, autotrascendencia y consumo de alcohol en jóvenes universitarios. J Health NPEPS. 2018; 3(1):5-21.

20. Porto NT, Ferreira DS, Figueiredo GLA. Comportamento de universitários em relação ao consumo de álcool, tabaco e outras drogas: subsídios para ações promotoras de saúde. Rev Educ Cult Contemp. 2019; 16(42):104-121.

21. Morales GA, Galera SAF, Reyes AT, Aguila SRG, Arroyo MLA, Castillo FAM. Factores de riesgo para el consumo de alcohol en adolescentes estudiantes. SMAD,
Rev Eletrônica Saúde Mental Álcool Drog. 2017; 13(1): 22-9

22. Silva CF, Oliveira AP, Mundim FL, Alves TA, Silva NA, Silva LLC, et al. Consumo de álcool e fatores de risco associados entre estudantes de uma Universidade Federal brasileira, 2014. Rev Bras Geo Med Saúde. 2016; 12(23):1-10.

23. Piedra $S$, Narváez $A$, Jácome $P$, Terán R, Barreto D, Aguirre R, et al. Consumo de riesgo de drogas utilizando la herramienta ASSIST modificado en estudiantes, docentes y personal administrativo de una universidad pública. Rev Med Vozandes. 2019; $30(2): 19-25$.

24. Morettl-Pires RO, Corradi-Webster CM. Adaptação e validação do Alcohol Use Disorder Identification Test (AUDIT) para população ribeirinha do interior da Amazônia, Brasil. Rio de Janeiro. Cad Saúde Pública. 2011; 27(3):497-509.

25. Monteiro LZ, Varela AR, Carneiro MLA, Alves LR, Góis RFG, Lima TB. Uso de tabaco e álcool entre acadêmicos da saúde. Rev Bras Promoç Saúde. 2018; 31(1):1-9.

26. Toledo L, Coutinho C, Bastos Fl. Panorama sobre a política de 
drogas e saúde mental no Brasil contemporâneo: prevenção e tratamento. Rio de Janeiro: Fundação Oswaldo Cruz; 2020.

27. El-Ansari W. Abdul Salam A, Suominen $S$. Is Alcohol Consumption Associated with Poor Perceived Academic Performance? Survey of Undergraduates in Finland. J Environ Res Public Health. 2020; 17(4):1369.

28. Gomes LS, Barroso CRD, Silvestre VA, Baylão ACP, Garcia SCM, Pacheco SJB. Consumo de álcool entre estudantes de medicina do Sul Fluminense - RJ. Rev Med (São Paulo). 2018; 97(3):260-6.
29. Finell LAC, Santos ARS, Ruas LF, Soares WD. Consumo de álcool por estudantes de engenharia biomédica. Rev Bionorte. 2017; $6(2): 1-12$

30. Mekonen T, Fekadu W, Chane T, Bitew S. Problematic Alcohol Use among University Students. Front Psychiatry. 2017; 8(86):1-5.

31. Silva EC, Tucci AM. Padrão de consumo de álcool em estudantes universitários (calouros) e diferença entre os gêneros. Temas Psicol. 2016; 24(1):313-323.

Conflito de interesses: Os autores declaram não haver conflito de interesses.

Participação dos autores:

- Concepção: Pereira ISSD, Melo RA, Gurgel AJP, Barbosa AGM, Zanatta IC, Melo SLA, Pereira JD.

- Desenvolvimento: Pereira ISSD, Melo RA, Gurgel AJP, Barbosa AGM, Zanatta IC, Melo SLA, Pereira JD.

- Redação e revisão: Pereira ISSD, Melo RA, Gurgel AJP, Barbosa AGM, Zanatta IC, Melo SLA, Pereira JD.

Como citar este artigo: Pereira ISSD, Melo RA, Gurgel AJP, Barbosa AGM, Zanatta IC, Melo SLA, et al. Uso de bebidas alcoólicas por estudantes de medicina. J Health NPEPS. 2020; 5(1):242-260.

Submissão: $27 / 02 / 2020$

Aceito: $15 / 05 / 2020$

Publicado: 01/06/2020 